\title{
Successful Factors of Flipped Classroom from the Perspective of Symbolic Interaction Theory
}

\author{
Lixin Wang ${ }^{1}$, Chonggang $\mathrm{Fu}^{2}$ \\ ${ }^{1)}$ School of Medium and Communication, Liaocheng University, Liaocheng, Shandong (lxwang@1cu.edu.cn) \\ ${ }^{2)}$ School of Chemistry and Chemical Engineering, Liaocheng University, Liaocheng, Shandong (cgfu@ @ lcu.edu.cn)
}

\begin{abstract}
Flipped classroom teaching (FCT) is emerging as an advanced pattern of instruction in recent years. With the increasing mature of multimedia and internet technology, FCT is widely accepted by teachers and students. But a variety of questions still exist with regard to FCT. Symbolic interaction theory is a micro-sociological and social psychological theory concerning the interactions of individuals in society. Teaching activity is also an interaction process between teaching participators through a variety of teaching symbols. FCT provides a favorable condition for the interaction of teaching symbols. Several successful factors involved in FCT are discussed from the perspective of symbolic interaction theory in the paper such as the preparation of high-quality video resources, standardization of an environment of self-learning for the students, activation of the classroom interaction, construction of an efficient interaction platform for the students and teacher.
\end{abstract}

Keywords—-symbolic interaction, flipped classroom, teaching resources, learning environment, interactive platform

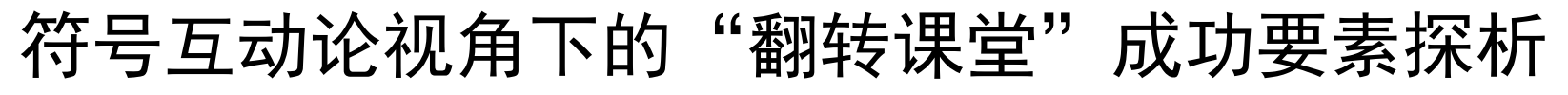

\author{
王立新 $^{1}$ 傅崇岗 $^{2}$ \\ 1) 聊城大学传媒技术学院, 聊城, 山东, 中国 \\ 2) 聊城大学化学化工学院, 聊城, 山东, 中国
}

摘 要 “翻转课堂”是近年来出现的一种先进的教学模式。随着多媒体技术和互联网技术的日渐成熟, 翻转课堂得到广大师生的 青崃。然而, 翻转课堂也存在着诸多问题。符号互动论是一种从微观层面关注社会中人与人之间互动的社会学和社会心理学理论, 教 学活动也是一种通过教学符号实现教学参与者各方互动的过程, 翻转课堂在一定程度上为教学符号互动创造了有利条件。本文以符号 互动论视角, 探讨了翻转课堂成功的诸多因素如精制教学资源、规范学生自主学习环境、激活课堂互动、构建师生交流平台。

关键词 符号互动, 翻转课堂, 教学资源, 学习环境, 交流平台

\section{1. 引言}

传统的教学方式以教师课堂知识传授和学生课后知识 内化两个阶段为特征, 有限的课堂时间被低效的知识灌输 所占用, 课堂交流时间缩短从而降低了学生知识内化的效 果。2011 年, 萨尔曼 - 可汗 (SalmanKhan) 在 TED 大会 上提出的全新教学方式一 “翻转课堂” (the Flipped Classroom) 为解决这个矛盾提供了契机, 完全符合中国《教 育信息化十年发展规划（2011-2020 年)》提出的 “教育信 息化的发展要以教育理念创新为先导, 以优质教育资源和 信息化学习环境建设为基础, 以学习方式和教育模式创新 为核心” ${ }^{[1]}$ 的思想, 引起了教育工作者的广泛关注。符号 互动理论是一种从微观层面关注社会中人与人之间的互动
的社会学理论, 其核心观点是: 人类创造与运用符号; 人 类通过识别他人使用的符号, 运用符号进行自我认识, 以 及对情境进行理解并作出反应, 发生人际之间的行动以及 这些行动的稳定模式与结构 ${ }^{[2]}$ 。教学活动正是教学参与者 各方通过教学符号互动的过程。同时, “翻转课堂” 作为一 种崭新的教学模式为教学互动提供了前所未有的学习情 境, 因此以符号互动理论视角研究 “翻转课堂” 教学模式 是必然的、有效的。

\section{2. “翻转课堂” 教学模式简介 \\ “翻转课堂” 的 “翻转” 是在教学程序上与传统的教 学模式比较而言的, 对学生来说主要包括课前通过视频接}


收教学内容的自主学习 (亦称知识传授) 阶段和课堂中通 过教师答疑、交流等手段的知识内化阶段。有些教学实践 中将学生课后知识升华阶段纳入教学过程而形成了包含三 阶段的 “翻转课堂” 教学模式 ${ }^{[3]}$ 。而传统教学模式的一般 教学流程是教师课前备课、课中教师讲学生听、课后学生 复习完成知识内化。翻转课堂将教师准备课程资源、学生 看视频接受教学内容的过程置于课前完成, 课堂时间交给 教师答疑解惑和学生彼此之间的交流。

除了形式上的改变, 教师和学生在教学过程中的角色 也发生了巨大变化。学生课堂上的被动听讲变成了课前的 我要看教学视频和课堂上的我要提问、我要发表意见建议。 老师从原来的课堂灌输者变成了知识的引领者, 课前用教 学资源吸引学生主动学习, 课中为学生的知识内化提供恰 当的课堂交流环境, 课后为学生知识升华、技能提升提供 辅导帮助。

\section{3. “翻转课堂” 教学模式研究现状及存在的问题}

翻转课堂的维形最早出现于 2007 年美国的 “林地公园 高中, 起初老师录制教学视频是为那些缺课的学生在家补 课之用。这种教学模式在 2011TED 大会之后很快得到认可 并推广。

中国学者对该教学模式的研究开始于 2012 年, 中国知 网期刊检索结果显示, 篇名中含有 “翻转课堂” 的研究论 文有 19 篇, 而 2013 年名称含有 “翻转课堂” 的论文达到 111 篇, 其受关注程度可见一斑。研究内容根据其关注点不 同可以分为三类: “翻转课堂” 教学模式在学科教学中的实 践、基于 “翻转课堂” 的教学平台设计、教学效果实证分 析。研究显示, “翻转课堂” 教学模式对创新教学理念、优 化教育资源、改进教学效果起到积极地推动作用。仅有的 三篇有关 “翻转课堂” 教学效果实证分析研究论文得到了 不尽相同的结论。雒真 ${ }^{[4]}$ 的研究认为 “翻转课堂在一定程 度上促进了 “现代教育技术” 实验教学”; 卢强 ${ }^{[3]}$ 的研究表 明, “翻转课堂并没有大幅度提高教学效果。仅仅做到形似 而非神似是教学实践效果没有明显提升的主要原因”; 马秀 麟等 ${ }^{[5]}$ 通过对现代信息技术教学实证研究后认为, 翻转课 堂更适合自主学习能力较强的学习者。

尽管学者们的研究结论各不相同, 但都对 “翻转课堂” 教学模式实施过程中面临的问题具有显而易见的共识: 第 一, 教学视频的质量是保证学生自主学习的关键; 第二, 学生自主学习能力是翻转课堂教学成功的基础; 第三, 良 好的课堂教学环境是课堂有效交流的保证; 但是, 如何从 根本上提高教学视频的质量、如何保证学生的自主学习效 果、如何才能创造高效课堂交流环境? 以符号互动论的视 角或许可以找到答案。

\section{4. 符号互动理论视角下的 “翻转课堂”}

教学活动是教学参与者各方通过教学符号进行互动的 过程。学生是教学的主体, 教学的最终目的是使学生的心 智得以成熟、情感得以发展、知识得以丰富、技能得以训 练。教学活动中可以使用公认的已有确定意义的教学符号, 在特定教学环境中也可以通过教学活动创造师生达成共识 的、便于交流教学信息的教学符号, 这就需要充分理解教 学符号的作用、体会教学环境、把握教学需求。依据教学 要素来分, 这些教学符号包括: 作为教学主导的教师符号、 作为教学主体的学生符号、作为教学中介的课程资源符号。 在当今的信息技术教学环境中, 教学资源形式丰富, 包括 文本、声音、视频、图片等, 通常以视频为主。在 “翻转 课堂” 教学模式下, 教学符号互动主要涉及四种互动形式: 教师符号与资源符号间的师本互动、学生符号与资源符号 间的生本互动、教师符号与学生符号间的师生互动和学生 符号间的生生互动, 前两者主要发生在课前知识传授阶段, 后两者主要发生在课堂中知识内化阶段。

\section{1 师本互动}

该阶段教师制作教学视频以备学生课前学习。教师制 作的教学视频作为重要的教学符号一资源符号是学生课前 接触学习内容的主要途径, 对学生最终的学习效果亦即教 学效果起着举足轻重的作用。师本互动通过教师符号对教 学内容的解读、重构形成适合学生符号认知水平的、与教 学目标相适应的、高效的教学视频。符号互动论认为, “人 们根据事物对于他们所具有的意义而对这些事物采取行 动”。因此, 使学生感受到教学视频中所含有的教师符号所 期望的教学意义, 是教师制作教学视频应该达到的最高境 界。

\section{2 生本互动}

生本互动发生在 “翻转课堂” 教学模式的重要阶段一 一自主学习阶段, 教师利用视频通过表意的姿态一符号来 传递教学信息, 学生通过与教学资源符号的互动完成知识 接收、理解。依据符号互动论, “事物的意义是通过遇到该 事物时所使用的解释过程来予以把握和修饰的, 事物的意 义不是一成不变的, 而是在解释的过程中根据特定的情景 来选择、审查和修正的意义 ${ }^{[6]}$ ”。学生通过观看教学视频学 习掌握教学内容, 教学视频的呈现方式、自主学习环境都 会影响其对教学内容的理解和把握。由此可知, 自主学习 阶段学生对教学视频的掌握程度取决于教学视频中教师符 号及资源符号表意的正确性以及学习环境。 


\section{3 师生互动与生生互动}

师生互动与生生互动是 “翻转课堂” 教学模式的核心, 此阶段教学各方均有参与, 是完成教学目标的关键一环。 教师以教师符号的形式呈现给学生, 而教师面对的是特性 各异的学生符号。师生互动中, 教师以恰当的符号形式讲 解课程内容、为学生答疑解惑, 同时接收来自学生符号的 反馈信息。学生符号的每个个体都可以接收来自教师符号 的信息。生生互动中, 学生符号可以接收来自其他学生符 号的信息, 同时作出反应。

符号互动论认为, 心灵的独特之处在于 (1) 以符号表 示环境中的客体;（2）预演对客体可选择的行动路线; (3) 抑制不合适的路线, 选择可公开采纳的行动途径。由此可 见, 学生会根据自己以往的经验预判自己的行动及语言会 产生何种效果, 然后采取对自己最有利的行动方式, 亦即 学生会根据教学环境来选择在师生互动、生生互动中的参 与方式。消极的课堂教学环境会抑制学生符号在师生互动 及生生互动中所采取的行动策略进而影响到教学内容的接 收与内化的效果。作为课堂主导的教师符号, 应该积极创 造良好的课堂情境, 为教学各方特别是学生一方积极准确 表达意见、顺利接受教学信息创造条件。

\section{5. “翻转课堂” 的成功要素}

卢强的研究已经表明, “仅仅做到形似而非神似” 并不 能使 “翻转课堂” 的教学效果想当然地优于传统教学模式。

“翻转课堂” 的成功需要关注以下几个要素:

\section{1 精制教学视频}

教学视频是教学内容的载体, 使教学视频起到 Zaid Ali Alsagoff 教授所说的 “激发、影响、告知” ${ }^{[7]}$ 作用是完成知 识传授的基本保证。可汗学院根据小学生注意力集中时长 大约十分钟的特点将视频控制在十分钟之内, 被证明是行 之有效的; 林地公园高中的教学视频是以两个老师对话的 方式呈现, 其中一位充当老师, 一位充当学生, 充当学生 的老师知道学生在哪些知识点的理解中会有困难, 他们在 对话中就会对这些问题进行讲解。这样的教学视频优势有 三: 第一, 容易引起学生的角色认同, 学生更容易融入其 中, 第二, 充当学生的老师通过发问替学生解决了看视频 过程中可能出现的疑问, 使自主学习得以顺利进行, 第三, 视频中的模拟问答一定程度上弥补了自主学习阶段师生互 动的缺失, 激发了学生的思维; 而得克萨斯州达拉斯地区 的生活学校的差异化教学视频使得所有学生都能接触到符 合自己认知水平的教学内容。这些成功的教学案例都是以 精心制作教学视频为保障的, 精制教学视频的关键在于适 应不同认知水平学生的需要。

\section{2 规范学生自主学习环境}

在 “翻转课堂” 教学模式中, 学生自主学习的物理空 间可以是自由选择的, 这就为教师监督调控学生自主学习 提出了挑战。充分使用现代信息技术手段构建规范统一的 网络自主学习平台, 不仅能够方便教师监控学生自主学习 状况，还可以提供师生、生生之间的实时或非实时互动, 同时又不妨碍自主学习物理空间的自由选择。

美国爱尔蒙湖小学等 ${ }^{[9]}$ 采用 moodle 教学平台规范了学 生的自主学习环境, 通过该平台教师可以了解学生是否观 看了教学视频、答题情况如何, 从而判断学生自主学习状 况, 给予针对性指导。学生也可以通过学习平台获得学习 资源, 自主解决学习中遇到的问题。由此看出, 学生自主 学习应该是在教师支持下的自主学习而不是放任自流, 既 使学生感受到自由学习的畅快又享受到教师适时的帮助。

\section{3 激活课堂互动}

有意义的学习是在互动中产生的。符号互动论认为, 人之所以成为独一无二的物种, 完全是互动的缘故。学生 在师生互动、生生互动中认识自我、了解他人、适应环境。 为了真正实现教师与学生的互动, 教师 “应尽可能调动多 种感官, 使用多种交流符号, 做到形神兼备, 声情并茂, 既要有丰富的教学语言, 又要借助抑扬顿挫的语调和丰富 的面部表情来增强语言的感染力和表现力, 使学生感觉听 课是一种美的享受。同时, 要善于调动学生的积极主动性, 让他们的思维和教师的思维互动起来, 引导他们将感觉和 视觉的注意力集中于教师的讲课中, 更好地在互动中完成 教学任务” ${ }^{[9]}$ 。研究表明,同类群体之间的相互影响远远 大 于教师和家长的影响 ${ }^{[10]}$,因此, 教师要注意引导学生之间的 积极互动, 创建有利于生生互动的课堂教学空间。

\section{4 构建师生交流平台}

教学过程是一个循序渐进、螺旋上升的过程。有些知 识点的学习并不是 “课前自主学习-课堂知识内化” 一个 循环就能完成的, 这可能需要两次甚至更多次重复。而教 学课时的限制使这些过程只能在课外完成。借助信息技术 构建的交流平台是课堂互动交流的延伸, 不仅可以规范学 生自主学习, 提供辅助学习资源, 还可以提供师生、生生 交流通道。同时, 也要注意学习资源质量与数量, 保证质 量的同时防止学生迷失在海量的资源中, 师生互动及时顺 畅但不干扰学生的自由, 使学生在宽松、方便的环境中在 教师有却似无的保驾护航中将学到的知识内化、升华。 


\section{参考文献(References)}

[1] Ministry of education of people's republic of china, "The national outline for medium and long term education reform and development (2010-2020)".

http://www.gov.cn/jrzg/2010-07/29/content_1667143.htm, 2010. 7.29

[2] L.F. Song, "Theory of western sociology", Nanjing: Nanjing university Press, 1997: 272.

[3] Q. Lu, "Reflection on the flipped classroom", E-education Research, 2013.8, pp.91-97.

[4] Z. Luo, "Exploratory optimization of experimental teaching in modern educational Technology in flipped classroom", Education Teaching Forum, 2013.10, pp.76-78.

[5] X. L. Ma, "An empirical study on the college public course of information technology", Distance Education Journal, 2013.1, pp. 79-85.
[6] Y. Song, H.C. Wang, "Some Inspirations of symbolic interactionism on construction of harmonious classroom", Humor and the Humorist, 2009.23, pp. 206.

[7] J. L. Zhang, "Analysis on the key factors of teaching pattern in flipped classroom”, Distance Education in China, 2013.10, pp. 59-64.

[8] Meris Stansbury. "A first-hand look inside a flipped classroom", http://www.eschoolnews.com/2012/02/09/a-first-hand-look-inside-a-flipped-classroom/, 2012-02-9.

[9] L. F. Song, "Symbolic interactionism and its Inspirations on education", Journal of Yangzhou University (Higher Education Study Edition), 2007.1, pp. 20-22.

[10] F. Liu, "Study on the situation definition of classroom teaching in view of symbolic interactionism”, Elementary \& Secondary Schooling Abroad, 2002.3, pp. 39-41. 